
\title{
Comparação do teste tuberculínico e do ensaio de liberação de interferon-gama para diagnóstico de tuberculose latente em agentes comunitários de saúde do Sul do Brasil, Rio Grande do Sul, 2012
}

doi: 10.5123/S1679-49742014000400009

\section{Comparison of tuberculin test and interferon-gamma release assay for diagnosing latent tuberculosis in Community Health Workers, State of Rio Grande do Sul, Brazil, 2012}

Paula Corrêa Machado

Curso de Farmácia, Universidade de Santa Cruz do Sul, Santa Cruz do Sul-RS, Brasil

Andréia Rosane de Moura Valim

Departamento de Biologia e Farmácia, Universidade de Santa Cruz do Sul e Programa de Pós-Graduação em Promoção da Saúde, Universidade de Santa Cruz do Sul, Santa Cruz do Sul-RS, Brasil

Ethel Leonor Noia Maciel

Programa de Pós-Graduação em Saúde Coletiva, Universidade Federal do Espírito Santo, Vitória-ES, Brasil

Thiago Nascimento do Prado

Programa de Pós-Graduação em Saúde Coletiva, Universidade Federal do Espírito Santo, Vitória-ES, Brasil

Tássia Silvana Borges

Programa de Pós-Graduação em Promoção da Saúde, Universidade de Santa Cruz do Sul, Santa Cruz do Sul-RS, Brasil

Alexandre Daronco

Curso de Medicina, Universidade de Santa Cruz do Sul, Santa Cruz do Sul-RS, Brasil

Marcelo Carneiro

Departamento de Biologia e Farmácia, Universidade de Santa Cruz do Sul, Santa Cruz do Sul-RS, Brasil

Lia Gonçalves Possuelo

Departamento de Biologia e Farmácia, Universidade de Santa Cruz do Sul e Programa de Pós-Graduação em Promoção da Saúde, Universidade de Santa Cruz do Sul, Santa Cruz do Sul-RS, Brasil

\section{Resumo}

Objetivo: comparar a capacidade de detecção de infecção latente por Mycobacterium tuberculosis em agentes comunitários de saúde (ACS) com uso do teste tuberculínico (TT) e do ensaio de liberação de interferon-gama (IGRA). Métodos: estudo transversal realizado no município de Santa Cruz do Sul, Rio Grande do Sul, Brasil, com aplicação do TT e do IGRA em 47 ACS no período de março a junho de 2012; o TT foi considerado positivo na presença de uma induração $\geq 10 \mathrm{~mm}$, assim como o IGRA, se houvesse concentração $\geq 0,35 \mathrm{UI} / \mathrm{ml}$ de interferon-gama. Resultados: 12 ACS apresentaram TT positivo e 6 tiveram IGRA positivo; a concordância entre os testes foi avaliada como pobre $(\kappa=0,063)$. Conclusão: apesar do número limitado de amostras, a alta discordância entre os testes evidencia a necessidade de desenvolver mais estudos que busquem encontrar uma explicação biológica para tais diferenças e avaliem a relação de custo-benefício na utilização do IGRA.

Palavras-chave: Tuberculose Latente; Pessoal de Saúde; Estudos Transversais, Teste Tuberculínico.

\begin{abstract}
Objective: to compare the ability to detect latent Mycobacterium tuberculosis infection among community bealth workers using the tuberculin skin test (TST) and Interferon-gamma release assay (IGRA). Methods: this was a cross-sectional study conducted in Santa Cruz do Sul-RS, Brazil, applying TST and IGRA to 47 community bealth workers between March and June 2012. TST was considered positive when induration was $\geq 10 \mathrm{~mm}$. IGRA was considered positive when interferon-gama concentration was at least $0.35 \mathrm{IU} / \mathrm{mL}$. Results: 12 community health workers presented positive TST and 6 had positive IGRA, the agreement between the tets was considered poor $(k=0.063)$. Conclusion: despite the limited number of samples there is a high discrepancy between the tests, what emphasize the need for more studies to find a biological explanation for these differences and to examine the cost-benefit of using IGRA.
\end{abstract}

Key words: Latent Tuberculosis; Health Personnel; Cross-sectional Studies, Tuberculin Test.

Endereço para correspondência:

Lia Gonçalves Possuelo - Universidade de Santa Cruz do Sul, Av. Independência, n 2293, Bairro Universitário, Santa Cruz do Sul-RS, Brasil. CEP: $96815-900$

E-mail:liapossuelo@unisc.br 


\section{Introdução}

Os profissionais de saúde encontram-se diretamente expostos ao Mycobacterium tuberculosis e são considerados uma população sob alto risco de infecção por esse agente. Uma revisão sistemática de estudos datados de 1945 a 2005, realizados com profissionais de saúde de países de média e baixa renda, mostrou uma prevalência média de 54\% de tuberculose (TB) latente entre esses trabalhadores; e concluiu que 0 maior risco de contrair a doença estava associado aos locais de trabalho e a algumas categorias profissionais. ${ }^{1}$

0 ambiente de trabalho da Saúde, geralmente, apresenta locais de passagem de muitos pacientes com TB. Alguns fatores, como falta de equipamentos de proteção individual, categoria profissional, tipo de atividades exercidas e tempo de exposição podem influenciar no contágio. ${ }^{2,3} 0$ cuidado dos pacientes com TB é uma das atividades realizadas pelos agentes comunitários de saúde (ACS), o que faz com que esses profissionais estejam frequentemente expostos ao $M$. tuberculosis. ${ }^{4}$

\section{0 cuidado dos pacientes com TB é uma das atividades realizadas pelos agentes comunitários de saúde (ACS), o que faz com que esses profissionais estejam frequentemente expostos ao M. tuberculosis.}

O diagnóstico precoce e 0 início imediato do tratamento são fundamentais para o controle da doença. ${ }^{5}$ Durante anos, o diagnóstico da infecção latente foi realizado apenas com uso do teste tuberculínico (TT), que mede a resposta de hipersensibilidade tardia a um derivado de proteína purificada (PPD) de mais de 200 antígenos do M. tuberculosis. ${ }^{6}$ No entanto, estudos demonstram que o TT apresenta limitações, entre elas a baixa especificidade, a possibilidade de ocorrência de falsos-positivos e a exposição a micobactérias não causadoras de tuberculose. ${ }^{6,7}$

Os ensaios de liberação de interferon-gama (IGRA) têm sido desenvolvidos como potenciais substitutos para o TT. Os IGRA realizam a quantificação in vitro da resposta imune celular pela detecção de interferongama, uma citocina liberada pelas células T, sensibi- lizadas pelo estímulo com antígenos específicos do M. tuberculosis. ${ }^{8}$ Esses testes apresentam inúmeras vantagens em relação ao TT: são mais sensíveis e específicos para detecção de tuberculose latente, fáceis de padronizar e implementar em laboratório, não expõem diretamente o paciente ao antígeno e permitem a inclusão de controles positivos e negativos. ${ }^{9}$ Normalmente, o resultado dos IGRA está disponível em 24 horas, ao contrário do TT, que necessita de 48 horas de espera para conhecimento do resultado. ${ }^{9}$

Muitos estudos têm mostrado o aumento do risco de infecção por M. tuberculosis entre profissionais de saúde ${ }^{1-4}$ porém, ainda não foram descritos trabalhos que comparassem a prevalência de TB latente entre ACS utilizando TT e IGRA. ${ }^{4}$

0 objetivo do presente estudo foi justamente comparar a capacidade de detecção de infecção latente por $M$. tuberculosis em agentes comunitários de saúde (ACS) com uso do TT e do IGRA.

\section{Métodos}

Foi realizado um estudo transversal, em que foram incluídos os ACS integrantes do Programa de Agentes Comunitários de Saúde (PACS) do município de Santa Cruz do Sul, estado do Rio Grande do Sul. No município, o PACS é coordenado por dois enfermeiros e executado por $60 \mathrm{ACS}$, distribuídos entre as zonas urbana e rural, com uma abrangência populacional de $11,4 \%$ e $8,7 \%$ respectivamente..$^{10}$

Santa Cruz do Sul-RS, cuja população somava 119.997 habitantes no ano de 2012, localiza-se na encosta inferior do nordeste do estado do Rio Grande do Sul (29 $43^{\prime} 59^{\prime \prime S}$ e $\left.52^{\circ} 24^{\prime} 52^{\prime \prime W}\right)$, a $155 \mathrm{~km}$ de sua capital, Porto Alegre-RS. Santa Cruz do Sul-RS é um dos 15 municípios prioritários para o controle da TB no estado. A incidência de tuberculose em Santa Cruz do Sul-RS no ano de 2011 foi de 40,3 por 100 mil habitantes. ${ }^{11}$ A coleta e análise dos dados foi realizada no período de março a junho de 2012.

Foram incluídos no estudo todos os profissionais maiores de 18 anos de idade, de ambos os sexos; foram excluídos aqueles que haviam realizado o TT no último ano. Os ACS foram convidados a participar de uma reunião em que lhes foram oferecidas informações sobre TB ativa e latente, além de apresentada a proposta do estudo. Em data previamente agendada, todos os ACS convidados e que aceitaram participar 
do estudo assinaram o Termo de Consentimento Livre e Esclarecido e, posteriormente, responderam a um questionário estruturado, aplicado individualmente, com perguntas sobre suas seguintes características pessoais:

a) sexo (feminino; masculino);

b) idade;

c) escolaridade (Ensino Fundamental; Ensino Médio; Ensino Superior);

d) tempo de trabalho na função ( $\leq 4$ anos; $>4$ anos);

e) exposição potencial a tuberculose no trabalho, na família e na comunidade;

f) uso de corticoides (Sim; Não);

g) tabagismo (Sim; Não);

h) uso de álcool (conforme critério CAGE) $;{ }^{12}$

i) presença da cicatriz da vacina com o bacilo de Calmette e Guérin, também conhecida por BCG (Sim; Não); e

j) uso de equipamentos de proteção individual (Sim; Não).

Após o preenchimento do questionário, foram coletados de cada paciente $3 \mathrm{ml}$ de sangue para a realização do IGRA (QuantiFERON ${ }^{\circledR}$-TB Gold In-Tube) e a aplicação do TT, conforme recomendado pelo 'Manual de Recomendações para o Controle de Tuberculose no Brasil'. ${ }^{33}$ A aplicação e a leitura do TT foram realizadas pelo mesmo profissional enfermeiro, devidamente treinado.

Para o IGRA, o total de $3 \mathrm{ml}$ de sangue foi distribuído em três tubos (controle negativo, controle positivo e antígenos específicos para M. tuberculosis - ESAT-6, CFP-10 e TB 7.7). As amostras foram incubadas com os antígenos estimuladores durante 24 horas, à temperatura de $37^{\circ} \mathrm{C}$. Separou-se o plasma dos tubos, os quais foram identificados de acordo com o material coletado. A quantidade de interferon-gama produzido foi medida por ensaio imunoenzimático (ELISA), de acordo com as instruções do fabricante (Cellestis Ltd, Carnegie, Victoria, Austrália). Os resultados foram analisados pelo software QuantiFERON ${ }^{\circledR}$-TB Gold, disponibilizado pelo fabricante.

Para o teste tuberculínico, foram considerados positivos aqueles que apresentaram induração $\geq 10 \mathrm{~mm},{ }^{13}$ e para 0 teste IGRA, o ponto de corte para resultados positivos foi uma concentração de pelo menos $0,35 \mathrm{UI} /$ $\mathrm{ml}$ de interferon-gama produzido após a estimulação com os antígenos específicos, conforme recomendação do fabricante. ${ }^{14}$ Todos os participantes com resultado positivo, independentemente do teste, foram enca- minhados para consulta com médico especialista no ambulatório de referência para TB do município de Santa Cruz do Sul-RS.

Após a coleta, os dados epidemiológicos e os resultados dos testes realizados foram digitados em planilha elaborada pelo programa Excel e posteriormente analisados pelo programa SPSS versão 17.0. A análise descritiva foi realizada a partir do cálculo de médias e do desvio-padrão, e frequências. A comparação das variáveis categóricas foi realizada utilizando-se o teste exato de Fisher. Adotou-se o nível de significância estatística $p \leq 0,05$. A concordância entre resultados dos testes TT e IGRA foi mensurada utilizando-se a estatística kappa ( $\kappa)$ : um valor de $\kappa<0,4$ foi considerado pobre; $\kappa>0,75$, concordância excelente; e um valor para $\kappa$ situado entre estes parâmetros, concordância boa. ${ }^{15}$ Os resultados do IGRA foram interpretados independentemente, sem o conhecimento dos resultados do TT.

Foram calculados a sensibilidade, a especificidade, $o$ valor preditivo positivo e o valor preditivo negativo do IGRA (o teste tuberculínico foi considerado o teste de referência). A sensibilidade foi calculada como a razão entre resultados positivos no IGRA e aqueles que tiveram TT positivo. A especificidade foi calculada como a razão entre resultados negativos no teste IGRA e aqueles que tiveram TT negativo. 0 valor preditivo positivo foi calculado como a proporção de participantes com resultados positivos no IGRA e no TT entre aqueles com resultado positivo no IGRA, e o valor preditivo negativo como a proporção de participantes com resultados negativos no IGRA e no TT e aqueles com resultado negativo no IGRA. A acurácia foi calculada como a razão entre o total de resultados corretos (soma dos verdadeiros positivos e verdadeiros negativos) e o total de participantes.

A pesquisa foi previamente submetida à apreciação do Comitê de Ética em Pesquisa da Universidade de Santa Cruz do Sul e aprovada sob o Parecer $n^{\circ}$ 2836/11.

\section{Resultados}

Dos 60 ACS atuantes no Programa de Agentes Comunitários de Saúde, 47 foram incluídos no estudo, e 13 considerados perdas por não terem comparecido à coleta das amostras e à entrevista. Entre os 47 indivíduos incluídos, 43 eram do sexo feminino. A média 
de idade dos participantes foi de 36,4 anos (desviopadrão=9,6 anos), sendo a idade mínima de 20 anos e a máxima de 53 anos. A média de tempo de trabalho dos profissionais como ACS foi de 7,3 anos, variando de 8 meses a 15 anos. Dos 47 ACS participantes, 46 relataram realizar visitas domiciliares diariamente e nunca utilizar qualquer tipo de equipamento de proteção individual. Quanto à vacinação com BCG, 45 eram vacinados.
0 T'T revelou 12 casos soropositivos para TB, enquanto o IGRA identificou 6. Observou-se baixa concordância entre os testes, com $\kappa=0,063$ (Tabela 1). A sensibilidade do IGRA foi de $16,7 \%$, a especificidade, de $88,6 \%$, o valor preditivo positivo, de $33,3 \%$, o valor preditivo negativo, de 75,6\%, e a acurácia, de 70,2\%.

Na Tabela 2, são descritos os resultados comparativos referentes aos fatores sociodemográficos e

Tabela 1 - Concordância entre ensaio de liberação de interferon-gama (IGRA) e o teste tuberculínico (TT) em agentes comunitários de saúde em Santa Cruz do Sul, Rio Grande do Sul, 2012

\begin{tabular}{lcc}
\hline \multirow{2}{*}{ IGRA } & \multicolumn{2}{c}{ TT } \\
\cline { 2 - 3 } & Positivo $(\geq 10 \mathrm{~mm})$ & Negativo $(<10 \mathrm{~mm})$ \\
\hline Positivo $(\geq 0,35 \mathrm{UI} / \mathrm{mL})$ & 2 & 4 \\
Negativo $(<0,35 \mathrm{UI} / \mathrm{mL})$ & 10 & 31 \\
\hline
\end{tabular}

Tabela 2 - Características dos agentes comunitários de saúde e resultado do teste tuberculínico (TT) e do ensaio de liberação de interferon-gama (IGRA) em Santa Cruz do Sul, Rio Grande do Sul, 2012

\begin{tabular}{|c|c|c|c|c|c|c|c|}
\hline \multirow{2}{*}{ Característica } & \multicolumn{2}{|c|}{ TT } & \multirow{2}{*}{$\mathbf{p}^{a}$} & \multicolumn{2}{|c|}{ IGRA } & \multirow{2}{*}{$\mathbf{p}^{a}$} & \multirow{2}{*}{$\mathrm{N}=47$} \\
\hline & Reator & Não reator & & Positivo & Negativo & & \\
\hline \multicolumn{8}{|l|}{ Idade (em anos) } \\
\hline $20-30$ & 5 & 14 & \multirow{4}{*}{0,084} & 2 & 17 & \multirow{4}{*}{$>0,999$} & 19 \\
\hline $31-40$ & 5 & 4 & & 1 & 8 & & 9 \\
\hline $41-50$ & 2 & 11 & & 2 & 11 & & 13 \\
\hline$>50$ & - & 6 & & 1 & 5 & & 6 \\
\hline \multicolumn{8}{|l|}{ Escolaridade } \\
\hline Ensino Fundamental completo & 2 & 8 & \multirow{3}{*}{0,328} & 1 & 9 & \multirow{3}{*}{0,141} & 10 \\
\hline Ensino Médio completo ou incompleto & 9 & 27 & & 4 & 32 & & 36 \\
\hline Ensino Superior incompleto & 1 & - & & 1 & - & & 1 \\
\hline \multicolumn{8}{|l|}{ Tempo de serviço (em anos) } \\
\hline$\leq 4$ & 2 & 13 & \multirow{2}{*}{0,288} & 1 & 14 & \multirow{2}{*}{0,648} & 15 \\
\hline$>4$ & 10 & 22 & & 5 & 27 & & 32 \\
\hline \multicolumn{8}{|l|}{ Vacina BCG } \\
\hline Sim & 12 & 33 & \multirow{2}{*}{$>0,999$} & 5 & 40 & \multirow{2}{*}{0,241} & 45 \\
\hline Não & - & 2 & & 1 & 1 & & 2 \\
\hline \multicolumn{8}{|l|}{ Uso de medicamento imunossupressor } \\
\hline Sim & 1 & 2 & \multirow{2}{*}{$>0,999$} & 1 & 2 & \multirow{2}{*}{0,343} & 3 \\
\hline Não & 11 & 33 & & 5 & 39 & & 44 \\
\hline \multicolumn{8}{|l|}{ Tabaco } \\
\hline Sim & 1 & 4 & \multirow{2}{*}{$>0,999$} & 1 & 4 & \multirow{2}{*}{0,511} & 5 \\
\hline Não & 11 & 31 & & 5 & 37 & & 42 \\
\hline \multicolumn{8}{|l|}{ Álcool } \\
\hline $1 \mathrm{vez} /$ semana & 5 & 16 & \multirow{3}{*}{$>0,999$} & 3 & 18 & \multirow{3}{*}{$>0,999$} & 21 \\
\hline$>1 \mathrm{vez} /$ semana & - & 1 & & - & 1 & & 1 \\
\hline Nunca & 7 & 18 & & 3 & 22 & & 25 \\
\hline \multicolumn{8}{|l|}{ Contato com paciente com tuberculose } \\
\hline Sim, no trabalho & 1 & 1 & \multirow{4}{*}{0,374} & - & 2 & & 2 \\
\hline Sim, na família & - & 2 & & - & 2 & 0,743 & 2 \\
\hline Sim, na comunidade & - & 5 & & 1 & 4 & 0,143 & 5 \\
\hline Não sabe & 11 & 27 & & 5 & 33 & & 38 \\
\hline
\end{tabular}

a) Valor p correspondente ao teste exato de Fisher 
clínicos associados com a tuberculose latente quando diagnosticada pelo TT e pelo IGRA. Não foi observada associação entre as variáveis 'idade', 'tempo de trabalho' e 'cicatriz da BCG' com o resultado do TT ou do IGRA.

\section{Discussão}

A concordância entre o TT e o IGRA encontrada neste estudo foi baixa $(k=0,063)$. Concordâncias pobres também foram observadas em outros estudos realizados com profissionais da Saúde. ${ }^{16,17}$ A discordância entre os testes ainda não é clara na literatura. Costa e cols. ${ }^{18}$ relatam que a vacinação repetida da BCG e o curto intervalo de tempo entre a vacinação e $o$ teste aumentam a discordância de TT'+/IGRA-. Outro estudo associa essa discordância (TT+/IGRA-) com o maior tempo de profissão, enquanto o TT-/IGRA+ foi associado ao contato com tuberculose na família. Nesta pesquisa, entretanto, esses fatores não foram relacionados com a discordância.

Apesar do elevado número de T'T+ entre os ACS, deve-se ressaltar as conhecidas limitações do TT, teste sujeito a eventual erro técnico na aplicação da injeção intradérmica. Quando essa aplicação é correta, produz uma pápula; porém, se a aplicação for demasiadamente profunda, o fluxo vascular pode diluir a PPD (proteína purificada derivada) e, possivelmente, apresentar um resultando errôneo. Outra desvantagem do TT' é a possibilidade de existência de falsos negativos, porquanto a ausência de reação em pacientes imunocomprometidos pode resultar em diminuição da sensibilidade do teste. ${ }^{19}$

Com relação ao IGRA, uma das desvantagens de seu uso é o elevado custo econômico, comparativamente ao TT. Há, também, relatos de altas taxas de resultados indeterminados e menor sensibilidade ao IGRA entre pessoas imunodeprimidas. ${ }^{20}$

Uma limitação do presente estudo foi a de não ter realizado testes para diagnóstico de doenças imunossupressoras, tais como a infecção pelo vírus da imunodeficiência humana, o HIV. Outras limitações deste estudo foram o número amostral pequeno e a perda de 13 ACS que não compareceram à coleta $\mathrm{e}$ às entrevistas.

Neste estudo, a prevalência de tuberculose latente encontrada pelo TT $(25,5 \%)$ foi semelhante à prevalência encontrada em mais um estudo realizado com profissionais da rede básica de saúde de Santa Cruz do Sul-RS, onde então, o percentual encontrado foi de $26,7 \% .{ }^{21}$ Em outro estudo, realizado com agentes comunitários de saúde do Espírito Santo, o percentual encontrado para a mesma prevalência foi de $26,7 \% .^{22}$ Já o resultado do IGRA demonstrou uma prevalência de $12,8 \%$, semelhante à encontrada entre profissionais da saúde lotados em hospitais na Alemanha e na Malásia. ${ }^{23,24}$ Pesquisas com o objetivo de avaliar a prevalência de tuberculose latente entre ACS utilizando IGRA ainda não foram realizadas.

o tempo de serviço e o contato com pacientes infectados também têm sido associado com infecção latente, em ambos os testes. ${ }^{25,26}$ No presente estudo, entretanto, essa relação não foi observada. Quanto ao tempo de serviço, a maioria dos profissionais participantes que foram reatores ao TT e positivos para 0 IGRA trabalhavam há mais de 4 anos como agentes comunitários de saúde. Costa e cols. ${ }^{18}$ verificaram que a probabilidade de um resultado IGRA ser positivo é maior com o aumento dos anos dedicados ao trabalho em saúde, enquanto que no TT, não se verificou tal associação. Segundo a pesquisa realizada por Oliveira e cols. ${ }^{21}$ os trabalhadores que referiam menos de 4 anos de tempo de exposição a pacientes com suspeita ou diagnóstico de tuberculose apresentavam uma taxa de positividade ao TT significativamente maior que aqueles com mais tempo de exposição.

No Brasil, ainda não foram desenvolvidos estudos utilizando o IGRA em ACS. No único estudo com o IGRA realizado no país e publicado, analisou-se a concordância entre o TT e o IGRA em contatos familiares de pacientes com tuberculose pulmonar e os testes por ele realizados mostraram concordância moderada $(\kappa=0,53){ }^{27}$

Quando se analisa qual dos testes seria o mais adequado à detecção de infecção latente, há divergências entre os dados descritos na literatura. Machado Jr. e cols. ${ }^{27}$ sugerem que $o$ Brasil deva continuar utilizando o TT, uma vez que o estudo por eles realizado, sobre uma região com alta prevalência de tuberculose latente e alvo de vacinação pela BCG, não observou influência da BCG nos resultados. Álvarez-Léon e cols. ${ }^{26}$ também concordam que hoje, seria precipitado substituir o TT pelo IGRA, salientando a importância de aprofundar pesquisas acerca da discordância entre os testes. Porém, vários estudos sugerem que os IGRA são mais específicos e deveriam ser utilizados para detecção de 
infecção por M. tuberculosis, devendo-se considerar, a priori, a prevalência de tuberculose na região, a taxa de vacinação, além de fatores operacionais e econômicos implicados. ${ }^{7,25,28}$

Como conclusão deste estudo, a concordância dos testes utilizados foi baixa, embora deva-se considerar o pequeno tamanho amostral. Novos estudos, sobre uma população maior e com diferentes características clínicas e sociodemográficas, devem ser realizados na tentativa de explicar tais diferenças e, ademais, avaliar o custo-benefício auferido com a utilização do novo teste - ensaio de liberação de interferon-gama, IGRA - em relação ao teste-padrão - teste tuberculínico, TT - utilizado no Brasil.

\section{Referências}

1. Josh R, Reingold AL, Menzies D, Pai M. Tuberculosis among health-care workers in low-and middleincome countries: a systematic review. PLoS Med. 2006 Dec;3(12):2376-91.

2. Faibis F, Castelain D, Moreau M. Étude de la prévalence de l'infection tuberculeuse chez le personnel soignant du service des urgences du centre hospitalier de Meaux par dosage de l'interféron gamma. Presse Med. 2011 dec;40(12 Part 1):516-20.

3. Oliveira SMVL, Honner MR, Paniago AMM, Aguiar ESA, Cunha RV. Prevalence of Mycobacterium tuberculosis among professionals in a university hospital, Mato Grosso do Sul, 2004. Rev Latino-Am Enferm. 2007 Nov-Dec;15(6):1120-4.

4. Moreira TR, Zandonade E, Maciel ELN. Risco de infecção tuberculosa em agentes comunitários de saúde. Rev Saude Publica. 2010 abr;44(2):332-8.

5. Oliveira MF, Arcêncio RA, Ruffino-Netto A, Scatena LM, Palha PF, Villa TCS. A porta de entrada para 0 diagnóstico da tuberculose no Sistema de Saúde de Ribeirão Preto/SP. Rev Esc Enferm. 2010 ago;45(4):898-904.

6. Lienhardt C, Fielding K, Hane AA, Niang A, Ndao CT, Karam F, et al. Evaluation of the prognostic value of IFN- $\gamma$ release assay and tuberculin skin test in household contacts of infectious tuberculosis cases in Senegal. PLoS Med. 2010 May;5(5):e10508.

7. Calayan V, AK O, Dabak G, Damadoglu E, Ketenci B, Ozdemir M, et al. Comparison of tuberculin skin testing and QuantiFERON-TB Gold-In Tube test in health care workers. Tuberk Toraks. 2011; 59(1):43-7.

\section{Contribuição dos autores}

Machado PC e Possuelo LG participaram da concepção e delineamento do estudo, análise e interpretação dos dados, redação e revisão crítica relevante do conteúdo intelectual do manuscrito.

Valim ARM participou da concepção e delineamento do estudo.

Maciel ELN, Prado TN, Borges TS e Daronco A participaram da redação e revisão crítica relevante do conteúdo intelectual do manuscrito.

Todos os autores aprovaram a versão final do manuscrito e declaram serem responsáveis por todos os aspectos do trabalho, garantindo sua precisão e integridade.

8. Sauzullo I, Masseti AP, Mengoni F, Rossi R, Lichtner M, Ajassa C, et al. Influence of previous tuberculin skin test on serial IFN- $\gamma$ release assays. Tuberculosis. 2011 Jul;91(4):322-6.

9. Naranjo JD, Carrillo CC, Calabuig MAG, Inmaculada SL. Estudio comparativo de QuantiFERON-TB Gold IT frente a tuberculina para el diagnóstico de la infección tuberculosa latente enestudios de contactos. Med Clin. 2011 sep;137(7):289-96.

10. Ministério da Saúde (BR). SIAB: Sistema de Informação da Atenção Básica [Internet]. Brasília: Datasus; c2008. [citado 2012 mar 28]. Disponível em: http://www2.datasus.gov.br/SIAB/index.php

11. Secretaria de Estado da Saúde (RS). Grupo de Trabalho Planejamento, Monitoramento e Avaliação da Gestão. Assessoria Técnica e de Planejamento. Plano Estadual de Saúde [Internet]. Rio Grande do Sul: Secretaria Estadual de Saúde; 2013. [citado 2014 out 29]. Disponível em: http://www.saude.rs.gov.br/ upload/1382374302_PES\%202012-2015\%20FINAL. pdf

12. Mayfield D, McLeod G, Hall P. The CAGE questionnaire: validation of a new alcoholism screening instrument. Am J Psychiatry. 1974 Oct;131(10):1121-3.

13. Ministério da Saúde (BR). Secretaria de Vigilância em Saúde. Departamento de Vigilância Epidemiológica. Manual de recomendações para o controle de tuberculose no Brasil [Internet]. Brasília: Ministério da Saúde; 2010 [citado 2012 jun 19]. Disponível em: http://www.cve.saude.sp.gov.br/htm/TB/mat_tec/ manuais/MS11_Manual_Recom.pdf 
14. Cellestis. QuantiFERON-TB Gold: método in tubo, teste de interferon-gama para sangue total, para medição de respostas aos péptidos antigénicos ESAT-6, CFP-10 \& TB7. 7 [Internet]. Hannover (DE): Cellestis; 2009 [citado 2013 fev 11]. Disponível em: http://www. cellestis.com/irm/content/pdf/PT\%20Portugal\%20 QFT\%20IT\%20insert\%20ver\%20C.pdf

15. Altman DG. Practical statistics for medical research. London: Chapman and Hall; 1991.

16. Lee K, Han MK, Choi HR, Choi CM, Oh YM, Lee SD, et al. Annual incidence of latent tuberculosis infection among newly employed nurses at a Tertiary Care University Hospital. Infect Control Hosp Epidemiol. 2009 Dec;30(12):1218-22.

17. Ringshausen FC, Nienhaus A, Schablon A, Schlosser S, Schultze-Werninghaus G, Rohde G. Predictor of persistently positive Mycobacterium tuberculosisspecific interferon-gamma responses in the serial testing of health care workers. BMC Infect Dis. 2010 Jul;10(220):1-11.

18. Costa JT, Sá R, Cardoso MJ, Silva R, Ferreira J, Ribeiro C, et al. Tuberculosis screening in Portuguese healthcare workers using the tuberculin skin test and the interferon- $\gamma$ release assay. Eur Respir J. 2009 Dec;34(6):1423-8.

19. Duarte R. Teste tuberculínico. Como optimizar? Rev Port Pneumol. 2009 mar;15(2):295-304.

20. Cattamanchi A, Ssewenyana I, Davis JL, Huang L, Worodria W, Boon SD, et al. Role of interferon gamma release assays in the diagnosis of pulmonary tuberculosis in patients with advanced HIV infection. BMC Infect Dis. 2010 Mar;10(75):1-7.

21. Oliveira JS, Possuelo LG, Severo K, Carneiro M, Krummenauer E, Machado CPH, et al. Avaliação da positividade ao teste tuberculínico entre os trabalhadores da rede básica de saúde. Rev Hosp Clin Porto Alegre. 2011;31(1):13-7.
22. Rodrigues PM, Moreira TR, Moraes AKL, Vieira RCA, Dietze R, Lima RCD, et al. Infecção por Mycobacterium tuberculosis entre agentes comunitários de saúde que atuam no controle da TB. J Bras Pneumol. 2009 abr;35(4):351-8.

23. Schablon A, Beckmann G, Harling M, Diel R, Nienhaus A. Prevalence of latent tuberculosis infection among health care workers in a hospital for pulmonary diseases. J Occup Med Toxicol. 2009 Jan;4(1):1-7.

24. Rafiza S, Rampal KG, Tahir A. Prevalence and risk factors of latent tuberculosis infection among health care workers in Malaysia. BMC Infect Dis. 2011 Jan;11(19):1-7.

25. Nienhaus A, Schablon A, Diel R. Interferon-gamma release assay for the diagnosis of latent TB infection analysis of discordant results, when compared to the tuberculin skin test. PLoS One. 2008 Jul;3(7):e2665.

26. Álvarez-Leon EE, Espinosa-Vega E, Santana-Rodríguez É, Molina-Cabrillana JM, Pérez-Arellano JL, Caminero JA, et al. Screening for tuberculosis infection in Spanish healthcare workers: comparison of the QuantiFERON-TB Gold in-tube test with the tuberculin skin test. Infect Control Hosp Epidemiol. 2009 Sep;30(9):876-83.

27. Machado JRA, Emodi K, Takenami I, Finkmoore BC, Barbosa T, Carvalho J, et al. Analysis of discordance between the tuberculin skin test and the interferongamma release assay. Int J Tuberc Lung Dis. 2009 Apr;13(4):446-53.

28. Girardi E, Angeletti C, Puro V, Sorrentino R, Magnavita $\mathrm{N}$, Vincenti $\mathrm{D}$, et al. Estimating diagnostic accuracy of tests for latent tuberculosis infection without a gold standard among healthcare workers. Euro Surveill. 2009 0ct;14(43):1-9.

Recebido em 25/04/2014 Aprovado em 01/09/2014 